\title{
DETECTING COHOMOLOGICALLY STABLE MAPPINGS
}

\author{
PHILIP L. BOWERS
}

\begin{abstract}
Let $f$ be a cohomologically stable mapping defined from a compactum $X$ to the $(n+1)\left[-\operatorname{cell} I^{n+1}\right.$, let $\pi: I^{n+1} \rightarrow I^{n}$ be the projection, and let $A=I^{n} \times\{1\}$ and $B=I^{n} \times\{-1\}$ be opposite faces of $I^{n+1}$. If $S$ is a separator or a continuum-wise separator of $f^{-1}(A)$ and $f^{-1}(B)$ in $X$, then $\pi f \mid S$ is cohomologically stable. This result is used to extend certain computations of cohomological dimension that are due to Walsh, who considered only the special case of the identity mapping on $I^{n+1}$.
\end{abstract}

1. Introduction. In [8], Walsh develops a method for computing the cohomological dimension of a class of spaces and shows that certain hereditarily strongly infinite dimensional subsets of the Hilbert cube have infinite cohomological dimension. These subsets of the Hilbert cube are constructed by intersecting continuumwise separators of opposite faces of the Hilbert cube (see [7 and 8] for a discussion of these constructions). While the various constructions can be done starting with any strongly infinite dimensional compactum, Walsh [8, Remark 7.1] notes that his techniques do not apply to this general setting. In this paper, we develop a method for detecting cohomological stability that can be used to extend the computation of cohomological dimension to a larger class of spaces than considered in [8]. The main results in [8] depend upon the fact that the identity map on the Hilbert cube is cohomologically stable and their proofs strongly depend upon the geometry of the Hilbert cube (thought of as the domain of the identity map). By contrast, we use techniques that depend upon the internal structure of specially constructed Eilenberg-Mac Lane complexes, permitting us to replace the identity map on the Hilbert cube by an arbitrary cohomologically stable mapping from any compactum to the Hilbert cube.

The paper is organized as follows. $\S 2$ contains the construction of special Eilenberg-Mac Lane complexes, $§ 3$ contains terminology, definitions, and basic facts and $\S 4$ contains the statements of the main results. In $\S 5$, the relationship between separators and continuum-wise separators is investigated and $\S 6$ contains proofs of the main results.

The author expresses his sincere thanks to J. J. Walsh for his helpful suggestions and kind criticisms.

2. Special $K(\mathbf{Z}, n)$ 's. Let $S^{n}$ denote an $n$-sphere. We use $K(\mathbf{Z}, n)$ to specify an Eilenberg-Mac Lane complex $X$ with $\pi_{i}(X)=\pi_{i}\left(S^{n}\right)$ for $i \leq n$ and $\pi_{i}(X)=0$ for $i \geq n+1$. The usual way to construct a $K(\mathbf{Z}, n)$ is to attach cells of dimension greater than or equal to $n+2$ to $S^{n}$. In this case, there is no obvious embedding of a

Received by the editors August 28, 1981.

1980 Mathematics Subject Classification. Primary 54F45, 55M10; Secondary 55P20.

Key words and phrases. Cohomological dimension, cohomologically stable mapping, EilenbergMac Lane space.

(C) 1982 American Mathematical Society 0002-9939/82/0000-0269/\$02.50 
$K(\mathbf{Z}, n-1)$ into this $K(\mathbf{Z}, n)$. We exploit the fact that a $K(\mathbf{Z}, n)$ can be constructed that in an essential manner contains not only an embedded $K(\mathbf{Z}, n-1)$, but also an embedded suspension of a $K(\mathbf{Z}, n-1)$. We construct such a $K(\mathbf{Z}, n)$ as follows.

For $n \geq 2$, let $K_{n-1}$ denote a specific $K(\mathbf{Z}, n-1)$ and let $\sum K_{n-1}$ denote its suspension. An easy Mayer-Vietoris argument shows that $H_{i}\left(\sum K_{n-1}\right)=0$ for $i \leq n-1$ and $H_{n}\left(\sum K_{n-1}\right)=\mathbf{Z}$. An application of the Hurewicz isomorphism theorem gives that $\pi_{i}\left(\sum K_{n-1}\right)=0$ for $i \leq n-1$ and $\pi_{n}\left(\sum K_{n-1}\right)=\mathbf{Z}$. Thus we can construct a $K(\mathbf{Z}, n)$ by attaching cells of dimension greater than or equal to $n+2$ to $\sum K_{n-1}$.

By starting with $S^{1}$ and applying the above construction successively, we get for each $n \geq 2$ an Eilenberg-Mac Lane complex with (i) $\pi_{i}\left(K_{n}\right)=\pi_{i}\left(S^{n}\right)$ for $i \leq n$ and $\pi_{i}\left(K_{n}\right)=0$ for $i \geq n+1$ and (ii) an inclusion $\sum S^{n-1} \subset \sum K_{n-1} \subset K_{n}$ inducing the isomorphism $\pi_{n}\left(\sum S^{n-1}\right) \stackrel{\sim}{=} \pi_{n}\left(K_{n}\right)$.

3. Definitions and basic concepts. By a space we mean a separable metric space, by a compactum we mean a compact space, and by a continuum we mean a connected compactum. We denote the (covering) dimension of a space $X$ by $\operatorname{dim} X$ and the integral cohomological dimension of a space $X$ by $c-\operatorname{dim} X$.

THEOREM 3.1 [4, p. 83]. For a space $X, \operatorname{dim} X \leq n$ if and only if for each closed subset $A$ and mapping $f: A \rightarrow S^{n}$ there is an extension $f: X \rightarrow S^{n}$.

THEOREM 3.2 [5, p. 7]. For a compactum $X, c-\operatorname{dim} X \leq n$ if and only if for each closed subset $A$ and mapping $f: A \rightarrow K_{n}$ there is an extension $\tilde{f}: X \rightarrow K_{n}$.

Let $B^{n+1}$ be an $(n+1)$-ball and denote its boundary by $S^{n}$. A mapping $f: X \rightarrow$ $B^{n+1}$ is unstable if there is a mapping $\bar{f}: X \rightarrow S^{n}$ with $\bar{f}=f$ on $f^{-1}\left(S^{n}\right)$; otherwise, $f$ is stable. By analogy with the above definition (noting the fact that $B^{n+1}$ is the cone of $S^{n}$ ), a mapping $f: X \rightarrow c K_{n}$ (the cone of $K_{n}$ ) is cohomologically unstable if there is a mapping $\bar{f}: X \rightarrow K_{n}$ with $\bar{f}=f$ on $f^{-1}\left(K_{n}\right)$; otherwise, $f$ is cohomologically stable. Since $B^{n+1} \subset c K_{n}$, we can speak of the cohomological stability of a mapping $f: X \rightarrow B^{n+1}$. Specifically, a mapping $f: X \rightarrow B^{n+1}$ is cohomologically unstable or stable if there is or is not, respectively, a mapping $\bar{f}: X \rightarrow K_{n}$ with $\bar{f}=f$ on $f^{-1}\left(S^{n}\right)$. The next results follow from Theorems 3.1 and 3.2 , respectively.

COROLlaRY 3.3. For a space $X, \operatorname{dim} X \geq n+1$ if and only if there is a stable mapping $f: X \rightarrow B^{n+1}$.

COROLlaRY 3.4. For a compactum $X, c-\operatorname{dim} X \geq n+1$ if and only if there is a cohomologically stable mapping $f: X \rightarrow c K_{n}$.

Let $Q=\prod\left\{I_{k}: k=1,2, \ldots\right\}$ denote the Hilbert cube where $I_{k}=[-1,1]$ and for each $n$ let $I^{n}=\prod\left\{I_{k}: k=1,2, \ldots, n\right\} \times(0,0, \ldots) \subset Q$. We say that a mapping $f: X \rightarrow Q$ is (cohomologically) stable provided $f \mid f^{-1}\left(I^{n}\right): f^{-1}\left(I^{n}\right) \rightarrow I^{n}$ is (cohomologically) stable for each $n$. Note that if $f: X \rightarrow Q$ is (cohomologically) stable, then $\pi f: X \rightarrow I^{n}$ is (cohomologically) stable where $\pi: Q \rightarrow I^{n}$ is the projection.

REMARK 3.5. Clearly, a cohomologically stable mapping into $B^{n+1}$ is stable. A standard example of a stable mapping which is not cohomologically stable is 
obtained by letting $\alpha: S^{k} \rightarrow S^{n}$ represent a nontrivial element of $\pi_{k}\left(S^{n}\right)$ with $k>$ $n$ and letting $f: B^{k+1} \rightarrow B^{n+1}$ be the "cone" of $\alpha$.

DEFINITION 3.6. Let $A$ and $B$ be disjoint closed subsets of a space $X$. A closed subset $S$ of $X$ is said to separate $A$ and $B$ in $X$ if $X \backslash S$ is the union of two disjoint open sets, one containing $A$ and the other containing $B$. A closed subset $S$ of $X$ is said to continuum-wise separate $A$ and $B$ in $X$ provided every continuum in $X$ from $A$ to $B$ meets $S$.

We will examine the relationship between separators and continuum-wise separators in $\S 5$. In the remainder of this section, $\Gamma$ denotes a finite or countably infinite indexing set.

DEFINITION 3.7. Let $X$ be a space. A family $\left\{\left(A_{k}, B_{k}\right): k \in \Gamma\right\}$ is essential in $X$ if, for each $k \in \Gamma,\left(A_{k}, B_{k}\right)$ is a pair of disjoint closed sets in $X$ such that if $S_{k}$ separates $A_{k}$ and $B_{k}$, then $\bigcap\left\{S_{k}: k \in \Gamma\right\} \neq \varnothing$.

A development of the theory of essential families can be found in [6, $\S 5]$.

EXAMPLE 3.8. Let $I^{\Gamma}=\prod\left\{I_{k}: k \in \Gamma\right\}$ where $I_{k}=[-1,1]$. For each $k \in \Gamma$, let $\pi_{k}: I^{\Gamma} \rightarrow I_{k}$ be the projection, and $A_{k}=\pi_{k}^{-1}(1)$ and $B_{k}=\pi_{k}^{-1}(-1)$. Then $\left\{\left(A_{k}, B_{k}\right): k \in \Gamma\right\}$ is an essential family in $I^{\Gamma}$.

The next result contains a precise statement of the relation between stable mappings and essential families.

Proposition 3.9 [8, Proposition 4.3]. Let $X$ be a compactum, let $\left\{\left(A_{k}^{\prime}, B_{k}^{\prime}\right)\right.$ : $k \in \Gamma\}$ be a family of pairs of disjoint closed subsets of $X$, and let $f_{k}: X \rightarrow I_{k}$ with $A_{k}^{\prime}=f_{k}^{-1}(1)$ and $B_{k}^{\prime}=f_{k}(-1)$. The family $\left\{\left(A_{k}^{\prime}, B_{k}^{\prime}\right): k \in \Gamma\right\}$ is essential if and only if the mapping $f: X \rightarrow I^{\Gamma}$ defined by $f=\left(f_{j}\right)_{j \in \Gamma}$ is stable.

4. Main results. Let $N$ denote an initial segment of the natural numbers (possibly all of the natural numbers) and let $I^{N}=\prod\left\{I_{k}: k \in N\right\}$ where $I_{k}=[-1,1]$. Thus, if $N$ is all of the natural numbers, $I^{N}$ is the Hilbert cube. For each $k \in N$, let $\pi_{k}: I^{N} \rightarrow I_{k}$ be the projection and let $A_{k}=\pi_{k}^{-1}(1)$ and $B_{k}=\pi_{k}^{-1}(-1)$. For a subset $N^{\prime}$ of $N$, let $\pi: I^{N} \rightarrow \prod\left\{I_{k}: k \in N^{\prime}\right\}$ be the projection. Given a mapping $f: X \rightarrow I^{N}$ defined on a compactum $X$, let $A_{k}^{\prime}=f^{-1}\left(A_{k}\right)$ and $B_{k}^{\prime}=f^{-1}\left(B_{k}\right)$, and for $Y \subset X$ let $f_{Y}=f \mid Y$. Observe that if $f$ is cohomologically stable (and therefore stable), Proposition 3.9 shows that $\left\{\left(A_{k}^{\prime}, B_{k}^{\prime}\right): k \in N\right\}$ is essential in $X$.

THEOREM 4.1. Let $X$ be compact, $f: X \rightarrow I^{N}$ be cohomologically stable, $\left\{N_{1}, N_{2}\right\}$ be a partition of $N$, and for each $k \in N$, let $S_{k}$ be a separator of $A_{k}^{\prime}$ and $B_{k}^{\prime}$ in $X$. If $Y=\bigcap\left\{S_{k}: k \in N_{1}\right\}$, then for each finite subset $\left\{t_{1}, \ldots, t_{q}\right\} \subset N_{2}$, $\pi f_{Y}: Y \rightarrow \prod\left\{I_{k}: k=t_{1}, \ldots, t_{q}\right\}$ is cohomologically stable. In particular, $c-\operatorname{dim} Y \geq$ $\operatorname{card}\left(N_{2}\right)$.

THEOREM 4.2. Let $N_{1}=\left\{r_{1}, r_{2}, \ldots\right\}$. The preceding theorem remains true if $S_{r_{1}}$ continuum-wise separates $A_{r_{1}}^{\prime}$ and $B_{r_{1}}^{\prime}$ in $X$ and, for $k \geq 2, S_{r_{k}} \cap S_{r_{k-1}}$ continuumwise separates $A_{r_{k}}^{\prime} \cap S_{r_{k-1}}$ and $B_{r_{k}}^{\prime} \cap S_{r_{k-1}}$ in $S_{r_{k-1}}$.

If $N=\{1,2, \ldots, n+1\}$, we denote $I^{N}$ by $I^{n+1}$. The main technical result used to prove Theorem 4.1 is the following proposition.

Proposition 4.3. Let $f: X \rightarrow I^{n+1}$ be a cohomologically stable mapping defined on a compactum $X$, let $S$ separate $A_{n+1}^{\prime}$ and $B_{n+1}^{\prime}$ in $X$, and let $\pi: I^{n+1} \rightarrow I^{n}$ be the projection. Then $\pi f_{S}: S \rightarrow I^{n}$ is cohomologically stable. 
Proposition 4.4. Proposition 4.3 remains true if $S$ continuum-wise separates $A_{n+1}^{\prime}$ and $B_{n+1}^{\prime}$ in $X$.

5. Separators and continuum-wise separators. This section investigates the relationship between separators and continuum-wise separators. While every separator is a continuum-wise separator, the reverse is not true; still, one can "approximate" arbitrarily closely any continuum-wise separator with a separator. It is this fact that allows us to prove Theorem 4.2 as a corollary of Proposition 4.3.

Throughout this section, $X$ denotes a compactum and $S$ denotes a continuumwise separator of the closed disjoint subsets $A$ and $B$ of $X$.

LEMMA 5.1. Suppose that $S \cap A=S \cap B=\varnothing$ and let $V$ be an open neighborhood of $S$. Then there exists a separator $S^{\prime}$ of $A$ and $B$ in $X$ contained in $V$.

Proof. Choose an open neighborhood $U$ of $S$ with $\bar{U} \subset V$ and $\bar{U} \cap A=\bar{U} \cap B=$ $\varnothing$. We claim that $S^{\prime}=\bar{U}$ separates $A$ and $B$ in $X$. Indeed, there is no connected set in $X \backslash U$ meeting both $A$ and $B$, for otherwise its closure is a continuum from $A$ to $B$ which does not meet $S$. This is enough to assure that $A$ and $B$ are separated in the compact set $X \backslash U$. Let $W^{\prime}$ be an open closed subset of $X \backslash U$ which contains $A$ and misses $B$. Then $W=W^{\prime} \cap(X \backslash \bar{U})$ is an open closed set in $X \backslash \bar{U}$ containing $A$ and missing $B$.

Proposition 5.2. Let $S$ be any continuum-wise separator of $A$ and $B$ in $X$ and let $V$ be an open neighborhood of $S$. Then there is a separator $S^{\prime}$ of $A$ and $B$ in $X$ contained in $V$.

ProOF. By the above lemma, all we need show is that there exist a continuumwise separator $S^{\prime \prime}$ of $A$ and $B$ in $X$ which is contained in $V$ and which has empty intersection with both $A$ and $B$.

Let $U$ be a neighborhood of $S$ with $\bar{U} \subset V$. For each positive integer $n$, let $N_{n}$ denote the open $(1 / n)$-neighborhood of $A$. We claim that there is an $n$ such that $\bar{U} \backslash N_{n}$ continuum-wise separates $A$ and $B$ (note that $\left(\bar{U} \backslash N_{n}\right) \cap A=\varnothing$ ). If no such $n$ exists, for each $n$ let $C_{n}$ be a continuum from $A$ to $B$ which does not meet $\bar{U} \backslash N_{n}$. Let $D_{n}$ be any component of $C_{n} \backslash N_{n}$ which meets $B$. Then $D_{n} \cap \bar{U}=\varnothing$ and $D_{n} \cap \bar{N}_{n} \neq \varnothing$ (for otherwise, $C_{n}$ is not connected). Thus, we obtain a sequence $\left\{D_{n}\right\}_{n=1}^{\infty}$ of nonempty continua in $X$ such that for each $n$, (i) $D_{n}$ meets $B$, (ii) the distance between $D_{n}$ and $A$ is less than or equal to $1 / n$, and (iii) $D_{n} \cap \bar{U}=\varnothing$. It is well known that the space of nonempty connected compact subsets of a compactum equipped with the Hausdorff metric is compact. Therefore, there is a nonempty continuum $D$ in $X$ and a subsequence $\left\{D_{n_{i}}\right\}$ of $\left\{D_{n}\right\}$ such that $D_{n_{i}} \rightarrow D$ in the Hausdorff metric. (i) implies that $D \cap B \neq \varnothing$, (ii) implies that $D \cap A \neq \varnothing$, and (iii) implies that $D \cap U=\varnothing$ and thus that $D \cap S=\varnothing$. This contradicts the fact that $S$ continuum-wise separates $A$ and $B$ in $X$.

Letting $M_{m}$ denote the $(1 / m)$-neighborhood of $B$, we let $S^{\prime \prime}$ be given by $\bar{U} \backslash\left(N_{n} \cup M_{m}\right)$ for appropriately chosen integers $n$ and $m$.

6. Proofs of main results. The proof of Proposition 4.3 mimics a standard proof of the result when "cohomologically stable" is replaced by "stable". The proof of the "stable" case uses the fact that $\sum S^{n-1}=S^{n}$ while the proof given below replaces this fact with a special property of the $K(\mathbf{Z}, n)$ 's constructed in $\S 2$, namely that $\sum S^{n-1} \subset \sum K_{n-1} \subset K_{n}$. 
ProOF OF PROPOSITION 4.3. We think of $I^{n}$ as $I^{n}=I^{n} \times\{0\} \subset I^{n} \dot{\times}$ $[-1,1]=I^{n+1}$ and $\pi$ as the usual retraction of $I^{n+1}$ onto $I^{n} \times\{0\}$. If $S$ separates $A_{n+1}^{\prime}$ and $B_{n+1}^{\prime}$ in $X$, then since $X$ is compact, $f(S)$ is a compact subset of $I^{n+1}$ which does not meet $A_{n+1}$ nor $B_{n+1}$. Choose $\epsilon>0$ so that $f(S) \subset$ $\pi_{n+1}^{-1}(-1+\epsilon, 1-\epsilon)$ where $\pi_{n+1}: I^{n+1} \rightarrow I_{n+1}$ is the projection. Let $j: I^{n+1} \rightarrow$ $I^{n+1}$ be the mapping which projects $\pi_{n+1}^{-1}[-1+\epsilon, 1-\epsilon]$ onto $\pi_{n+1}^{-1}(0)=I^{n}$ and takes $\left(\pi_{n+1}^{-1}[-1,-1+\epsilon), \pi_{n+1}^{-1}(1-\epsilon, 1]\right)$ homeomorphically onto $\left(\pi_{n+1}^{-1}[-1,0)\right.$, $\left.\pi_{n+1}^{-1}(0,1]\right)$. We show that $j f: X \rightarrow I^{n+1}$ is cohomologically stable. Choose $\eta$ so that $\eta$-close maps into $S^{n}$ are homotopic in $S^{n}$ and choose a homeomorphism $h: I^{n+1} \rightarrow I^{n+1}$ so that $h$ is $\eta$-close to $j$ (and therefore $h f$ is $\eta$-close to $j f$ ). The composition $h f$ is cohomologically stable since $f$ is and since $h$ is a homeomorphism. Since $h f \mid f^{-1}\left(S^{n}\right)$ and $j f \mid f^{-1}\left(S^{n}\right)$ are $\eta$-close, they are homotopic as maps into $S^{n}$. Thus if $j f$ is not cohomologically stable, then the homotopy extension theorem implies that $h f$ is not cohomologically stable, a contradiction. Now since $\pi j f_{S}=$ $\pi f_{S}$, all we need show is that $\pi j f_{S}$ is cohomologically stable. Thus, since $j f(S) \subset I^{n}$ and $j f$ is cohomologically stable, if we prove 4.3 in the special case that $f(S) \subset I^{n}$, we are done.

Assume that $f(S) \subset I^{n}$ and thus $\pi f_{S}=f_{S}$. We think of $S^{n}$ as $\partial I^{n+1}=S^{n}=$ $\sum S^{n-1} \subset \sum K_{n-1}$. Suppose $\pi f_{S}=f_{S}$ is not cohomologically stable. Then there is a mapping $\bar{f}: S \rightarrow K_{n-1}$ with $\bar{f}=f_{S}$ on $f_{S}^{-1}\left(S^{n-1}\right)=f^{-1}\left(S^{n}\right) \cap S$. Let $c_{+}$and $c_{-}$ denote the suspension points of $\sum K_{n-1}$ and let $g^{\prime}$ be the mapping on $S \cup A_{n+1}^{\prime} \cup$ $B_{n+1}^{\prime}$ into $\sum K_{n-1}$ given by $g^{\prime}|S=\bar{f}| S, g^{\prime}\left(A_{n+1}^{\prime}\right)=c_{+}$, and $g^{\prime}\left(B_{n+1}^{\prime}\right)=c_{-}$. Use the fact that $S$ separates $A_{n+1}^{\prime}$ and $B_{n+1}^{\prime}$ in $X$ and that the upper and lower cones of $\sum K_{n-1}$ are AR's to extend $g^{\prime}$ to a mapping $g: X \rightarrow \sum K_{n-1} \subset K_{n}$. Observe that $\sum K_{n-1} \backslash\left\{c_{+}\right\}$and $\sum K_{n-1} \backslash\left\{c_{-}\right\}$are contractible and use this along with the fact that $f\left|f^{-1}\left(S^{n}\right) \cap S=g\right| f^{-1}\left(S^{n}\right) \cap S$ to show that $f \mid f^{-1}\left(S^{n}\right)$ and $g \mid f^{-1}\left(S^{n}\right)$ are homotopic rel $f^{-1}\left(S^{n}\right) \cap S$ as maps into $\sum K_{n-1} \subset K_{n}$. Since $g \mid f^{-1}\left(S^{n}\right)$ extends and $K_{n}$ is an ANE, the homotopy extension theorem applies to give an extension of $f \mid f^{-1}\left(S^{n}\right)$ into $K_{n}$, a contradiction of the fact that $f$ is cohomologically stable.

PROOF OF PROPOSITION 4.4. Suppose $\pi f_{S}$ is not cohomologically stable. Let $\bar{f}: S \rightarrow K_{n-1}$ be a mapping with $\bar{f}=\pi f_{S}$ on $\left(\pi f_{S}\right)^{-1}\left(S^{n-1}\right)$. Use $\pi f$ to extend $\bar{f}$ to $S \cup(\pi f)^{-1}\left(S^{n-1}\right)$ and let $g$ be an extension of this to a neighborhood $U$ of $S \cup(\pi f)^{-1}\left(S^{n-1}\right)$. Let $S^{\prime}$ be a separator of $A_{n+1}^{\prime}$ and $B_{n+1}^{\prime}$ contained in $U$. Then $\pi f_{S^{\prime}}$ is not cohomologically stable since $g_{S^{\prime}}: S^{\prime} \rightarrow K_{n-1}$ and $g_{S^{\prime}}=\pi f_{S^{\prime}}$ on $\left(\pi f_{S^{\prime}}\right)^{-1}\left(S^{n-1}\right)=f^{-1}\left(S^{n}\right) \cap S^{\prime}$. This contradicts Proposition 4.3.

REMARK 6.1. Since $X$ continuum-wise separates $A_{n+1}$ and $B_{n+1}, \pi f: X \rightarrow$ $I^{n}$ is cohomologically stable. Using this observation one can easily prove the following result (which will be used in the proof of Theorem 4.1): let $f: X \rightarrow$ $I^{n}$ be cohomologically stable and let $\left\{t_{1}, \ldots, t_{q}\right\} \subset\{1, \ldots, n\}$. Then $\pi f: X \rightarrow$ $\prod\left\{I_{k}: k=t_{1}, \ldots, t_{q}\right\}$ is cohomologically stable where $\pi$ is the projection $I^{n} \rightarrow$ $\prod\left\{I_{k}: k=t_{1}, \ldots, t_{q}\right\}$.

Proof OF THEOREM 4.1. If $N$ is finite, Theorem 4.1 is, by induction, a direct consequence of Proposition 4.3 and Remark 6.1. If $N$ is infinite, the proof of the theorem is similar to that in $[8, \S 6]$. The proof goes as follows.

For convenience we assume that $\left\{t_{1}, t_{2}, \ldots, t_{q}\right\}=\{1,2, \ldots, q\}$ in the statement of Theorem 4.1. If $\pi f_{Y}$ is not cohomologically stable, then there exists a mapping $\bar{f}: Y \rightarrow K_{q-1}$ with $\bar{f}=\pi f_{Y}$ on $\left(\pi f_{Y}\right)^{-1}\left(S^{q-1}\right)$; extend $\bar{f}$ to $Y \cup(\pi f)^{-1}\left(S^{q-1}\right)$ 
using $\pi f$ and let $\tilde{f}: U \rightarrow K_{q-1}$ be an extension of $\bar{f}$ to a neighborhood $U$ of $Y U$ $(\pi f)^{-1}\left(S^{q-1}\right)$. Let $\left\{r_{1}, \ldots, r_{s}\right\} \subset N_{1}$ be such that $\bigcap\left\{S_{k}: k=r_{1}, \ldots, r_{s}\right\} \subset U$; let $n=\max \left\{r_{1}, \ldots, r_{s}\right\}$ and for each $r_{i}$, let $S_{r_{i}}^{\prime}=S_{r_{i}} \cap f^{-1}\left(I^{n}\right)\left(I^{n}=I^{n} \times(0,0, \ldots) \subset\right.$ $\left.I^{N}\right)$ and let $Y^{\prime}=\bigcap\left\{S_{r_{i}}^{\prime}: i=1, \ldots, q\right\}$. Let $g=f \mid f^{-1}\left(I^{n}\right)$. Then $g: f^{-1}\left(I^{n}\right) \rightarrow I^{n}$ is cohomologically stable and the case for $N$ finite shows that $\pi g_{Y^{\prime}}$ is cohomologically stable. However, $\tilde{f}_{Y^{\prime}}: Y^{\prime} \rightarrow K_{q-1}$ and $\tilde{f}_{Y^{\prime}}=\pi f_{Y^{\prime}}=\pi g_{Y^{\prime}}$ on $\left(\pi f_{Y^{\prime}}\right)^{-1}\left(S^{q-1}\right)=$ $\left(\pi g_{Y^{\prime}}\right)^{-1}\left(S^{q-1}\right)$. This says that $\pi g_{Y^{\prime}}$ is cohomologically unstable, a contradiction.

PROOF OF THEOREM 4.2. Theorem 4.2 is proved in the same way that Theorem 4.1 is except that Proposition 4.4 is used in place of Proposition 4.3.

\section{REFERENCES}

1. R. H. Bing, $A$ hereditarily infinite-dimensional space, General Topology and its Relation to Modern Analysis and Algebra. II (Proc. Second Prague Topological Sympos., 1966), Academia, Prague, 1967, pp. 56-62.

2. D. W. Henderson, An infinite-dimensional compactum with no positive-dimensional compact subset-a simpler construction, Amer. J. Math. 89 (1967), 105-121.

3. $\ldots$ Each strongly infinite-dimensional compactum contains a hereditarily infinite-dimensional compact subset, Amer. J. Math. 89 (1967), 122-123.

4. W. Hurewicz and H. Wallman, Dimension theory, Princeton Univ. Press, Princeton, N.J., 1941.

5. V. I. Kuz'minov, Homological dimension theory, Russian Math. Surveys 23 (1968), no. 5, 1-45.

6. L. Rubin, R. Schori and J. Walsh, New dimension-theory techniques for constructing infinitedimensional examples, General Topology and Appl. 10 (1979), 93-102.

7. R. Schori and J. Walsh, Examples of hereditarily strongly infinite-dimensional compacta, Topology Proc. 3 (1978), 495-506.

8. J. J. Walsh, A class of spaces with infinite cohomological dimension, Michigan Math. J. 27 (1980), 215-222.

9. __ Infinite dimensional compacta containing no $n$-dimensional $(n \geq 1)$ subsets, Topology 18 (1979), 91-95.

10. A. V. Zarelua, On hereditarily infinite dimensional spaces, Theory of Sets and Topology (Memorial volume in honor of Felix Hausdorff), edited by G. Asser, J. Glachsmeyer and W. Rinow, VEB Deutscher Verlag der Wissenschaften, Berlin, 1971, pp. 509-525. (Russian)

11. _ Construction of strongly infinite-dimensional compacta by means of rings of continuous functions, Soviet Math. Dokl. 15 (1974), 106-110.

Department of Mathematics, University of Tennessee, KNOXVille, TennesSEE 37916 